Pacific

Journal of

Mathematics

ASYMPTOTIC BEHAVIOR OF THE KOBAYASHI METRIC ON CONVEX DOMAINS

LINA LEE

Volume $238 \quad$ No. 1

November 2008 


\title{
ASYMPTOTIC BEHAVIOR OF THE KOBAYASHI METRIC ON CONVEX DOMAINS
}

\author{
LINA LEE
}

\begin{abstract}
We calculate estimates for invariant metrics on a finite type convex domain in $\mathbb{C}^{n}$ using the Sibony metric. We also discuss a possible modification of the Sibony metric.
\end{abstract}

\section{Introduction}

The Kobayashi metric $F(P, \xi)$ on a domain $\Omega \subset \mathbb{C}^{n}$ at a point $P \in \Omega$ in the direction $\xi \in T_{P}(\Omega)$ is defined as

$$
F(P, \xi)=\inf \left\{\alpha>0: \exists \phi \in \Omega(\mathbb{D}), \phi(0)=P, \phi^{\prime}(0)=\xi / \alpha\right\},
$$

where $\Omega(\mathbb{D})$ denotes the family of holomorphic mappings from the unit disc $\mathbb{D}$ in $\mathbb{C}$ to $\Omega$. It is known that the Kobayashi metric is greater than any biholomorphically invariant metric $G$ that satisfies the following properties:

(1) $G^{\mathbb{D}}: \mathbb{D} \times \mathbb{C} \rightarrow \mathbb{R}^{+} \cup\{0\}$ coincides with the Poincare metric on the unit disc in $\mathbb{C}$;

(2) $G$ is nonincreasing under holomorphic mappings, that is, if $\Phi: \Omega \rightarrow \tilde{\Omega}$ is a holomorphic mapping and $P \in \Omega, \xi \in T_{P}(\Omega)$, then

$$
G^{\Omega}(P, \xi) \geq G^{\tilde{\Omega}}\left(\Phi(P), \Phi_{*}(P) \xi\right) .
$$

It has been of importance to study the asymptotic behavior of the Kobayashi metric near the boundary of a holomorphically convex domain. Several authors have proved results on pseudoconvex domains: Ian Graham [1975] proved a result on a strongly pseudoconvex domain and David Catlin [1989] studied the behavior on a weakly pseudoconvex domain in $\mathbb{C}^{2}$.

As we can see from the definition of the Kobayashi metric (1-1), the difficulty in estimating the Kobayashi metric lies in finding the lower estimate since the upper estimate can be found rather easily by constructing one analytic disc in $\Omega$ that satisfies the desired properties.

MSC2000: 32F45, 32F18.

Keywords: Kobayashi metric, convex domain, Sibony metric. 
Graham [1975] calculated the metric explicitly on ellipsoids and found the estimate on a strongly pseudoconvex domain by approximating it with ellipsoids and proving results on localization of the metric. Catlin [1989] proved the result by estimating Carathéodory metric $F_{C}(P, \xi)$, which is defined as

$$
F_{C}(P, \xi)=\sup \left\{\left|f_{*}(P) \xi\right|=\left|\sum_{i=1}^{n} \frac{\partial f(P)}{\partial z_{i}} \xi_{i}\right|: f \in \mathbb{D}(\Omega), f(P)=0\right\} .
$$

The Carathéodory metric satisfies above two properties and hence is less than the Kobayashi metric. So one can estimate the Carathéodory metric and find a lower estimate for the Kobayashi metric.

In this paper, we estimate the Kobayashi metric on a convex domain in $\mathbb{C}^{n}$ using the Sibony metric, whose definition can be found in Section 2. The Sibony metric also satisfies the two properties above and hence gives a lower estimate for the Kobayashi metric. The advantage of using the Sibony metric over using the Carathéodory metric is that the Sibony metric uses bounded plurisubharmonic functions whereas the Carathéodory metric uses bounded holomorphic functions, which are usually more difficult to construct than plurisubharmonic functions. In Section 2, we give a more detailed explanation of the Sibony metric.

We assume $\Omega=\{\rho<0\} \Subset \mathbb{C}^{n}$ is a smoothly bounded convex domain,

$$
P \in \partial \Omega, \quad v=\frac{\nabla \rho(P)}{\|\nabla \rho(P)\|} \quad \text { and } \quad P_{\delta}=P-\delta \nu \in \Omega .
$$

For

$$
\xi \in T_{P}^{\mathbb{C}}(\partial \Omega)=T_{p}(\partial \Omega) \cap J T_{P}(\partial \Omega),
$$

where $J$ is the standard complex structure of $\mathbb{C}^{n}$, we define $\Delta(\partial \Omega, P, \xi)$ as the tangency of the $\partial \Omega$ at $P$ in the direction $\xi$, that is,

$$
\Delta(\partial \Omega, P, \xi)=v_{0}(\rho(P+\xi z)), \quad \text { for } z \in \mathbb{C},
$$

where $v_{0}(f(z))$ denotes the vanishing order of $f$ at $z=0$.

Lempert [1981] proved that the Kobayashi metric and the Carathéodory metric coincide on a convex domain in $\mathbb{C}^{n}$. Hence the Sibony metric also coincides with the Carathéodory metric and the Kobayashi metric. Let us denote the (Kobayashi or Sibony or Carathéodory) metric as $F(Q, \xi)$ for $Q \in \Omega$ and $\xi \in T_{Q}(\Omega)$. Then we have the following theorem.

Theorem 1. If $\Omega \subset \mathbb{C}^{n}$ is a smoothly bounded convex domain of finite type, then we have

$$
F\left(P_{\delta}, \xi\right) \approx \frac{|\xi|}{\delta^{1 / m}}, \quad \xi \in T_{P}^{\mathbb{C}}(\partial \Omega)
$$


where $m=\Delta(\partial \Omega, P, \xi)$, and

$$
F\left(P_{\delta}, v\right) \approx \frac{1}{\delta}
$$

for all sufficiently small $\delta>0$.

Remark 1. In (1-3) and (1-4), the notation " $\approx$ " means that there exist positive constants $c, C, c^{\prime}$ and $C^{\prime}$ that do not depend on $\delta$ such that

$$
c \frac{|\xi|}{\delta^{1 / m}} \leq F\left(P_{\delta}, \xi\right) \leq C \frac{|\xi|}{\delta^{1 / m}} \quad \text { and } \quad c^{\prime} \frac{1}{\delta} \leq F\left(P_{\delta}, v\right) \leq C^{\prime} \frac{1}{\delta},
$$

for all $\delta>0$ sufficiently small.

The boundedness from above can be easily shown using the definition of the Kobayashi metric. We can express the defining function using the Taylor series and find an analytic disc that has the proper size in the estimating direction. For more details, see [Lee 2007]. In Section 2, we prove the boundedness from below.

We also prove the following theorem.

Theorem 2. Suppose $\Omega \Subset \mathbb{C}^{n}$ is a smoothly bounded convex domain. Let

$$
X=a v+b T,
$$

where $T \in T_{P}(\Omega)$ and $a, b>0$. Then we have

$$
F\left(P_{\delta}, X\right) \geq \frac{|a|}{6 \delta} .
$$

In Section 2, we give a brief background of invariant metrics and finite type and, in Section 3, we prove Theorem 1 and Theorem 2. In Section 4, we discuss a possible modification of the Sibony metric.

\section{Background: invariant metrics and the concept of finite type}

We say $F: T \Omega \rightarrow \mathbb{R}^{+} \cup\{0\}$, is an invariant metric if $F$ is invariant under biholomorphic mappings, that is, if $\Phi: \Omega_{1} \rightarrow \Omega_{2}$ is a biholomorphic mapping between $\Omega_{1}$ and $\Omega_{2}$ and $P \in \Omega_{1}, \xi \in T_{P}\left(\Omega_{1}\right)$, then

$$
F^{\Omega_{1}}(P, \xi)=F^{\Omega_{2}}\left(\Phi(P), \Phi_{*}(P) \xi\right) .
$$

For example, the Poincaré metric $P(z, \xi)$ on the unit disc $\mathbb{D}$ in $\mathbb{C}$, which is defined as

$$
P(z, \xi)=\frac{|\xi|}{1-|z|^{2}},
$$

is invariant under automorphisms of the unit disc. 
Two possible generalizations of the Poincaré metric to an arbitrary domain $\Omega$ in $\mathbb{C}^{n}$ are the Kobayashi metric, $F_{K}(P, \xi)$, and the Carathéodory metric, $F_{C}(P, \xi)$, which are defined as

$$
\begin{aligned}
& F_{K}(P, \xi)=\inf \left\{\alpha: \exists \phi \in \Omega(\mathbb{D}), \phi(0)=P, \phi^{\prime}(0)=\xi / \alpha, \alpha>0\right\}, \\
& F_{C}(P, \xi)=\sup \left\{\left|f_{*}(P) \xi\right|=\left|\sum_{i=1}^{n} \frac{\partial f(P)}{\partial z_{i}} \xi_{i}\right|: f \in \mathbb{D}(\Omega), f(P)=0\right\},
\end{aligned}
$$

where $A(B)$ denotes the family of holomorphic mappings from $B$ to $A$ and $\mathbb{D}$ the unit disc in $\mathbb{C}$.

The Kobayashi metric is the largest pseudometric and the Carathéodory metric is the smallest in the following sense.

Proposition 1. Suppose that

$$
\tilde{F}^{\Omega}: T \Omega \rightarrow \mathbb{R}^{+} \cup\{0\}
$$

is a pseudometric on $\Omega$ such that $\tilde{F}^{\mathbb{D}}$ coincides with the Poincaré metric and $\tilde{F}$ is nonincreasing under holomorphic mappings, that is, if $\Phi: \Omega_{1} \rightarrow \Omega_{2}$ is a holomorphic mapping and $P \in \Omega_{1}$, then we have

$$
\tilde{F}^{\Omega_{1}}(P, \xi) \geq \tilde{F}^{\Omega_{2}}\left(\Phi(P), \Phi_{*}(P) \xi\right), \quad \text { for all } \xi \in T_{P}^{\mathbb{C}}\left(\Omega_{1}\right) .
$$

Then we always have

$$
F_{C}^{\Omega}(P, \xi) \leq \tilde{F}^{\Omega}(P, \xi) \leq F_{K}^{\Omega}(P, \xi) .
$$

The Sibony metric is defined as follows.

Definition 1 (Sibony metric). Let $\Omega \in \mathbb{C}^{n}$ be a domain and $P \in \Omega$. We define a set of functions, $A_{\Omega}(P)$, such that $u \in A_{\Omega}(P)$ if and only if

(1) $u$ is $C^{2}$ near $P$;

(2) $u(P)=0$;

(3) $0 \leq u(z) \leq 1$ for all $z \in \Omega$;

(4) $\log u$ is plurisubharmonic on $\Omega$.

We define the infinitesimal Sibony metric $F_{\Omega}^{S}$ at $P$ in the direction $\xi \in \mathbb{C}^{n}$ as

$$
F_{S}(P, \xi) \equiv \sup _{u \in A_{\Omega}(P)}\left(\sum_{i, j=1}^{n} \frac{\partial^{2} u}{\partial z_{i} \partial \bar{z}_{j}}(P) \xi_{i} \bar{\xi}_{j}\right)^{1 / 2} .
$$

The Sibony metric coincides with the Poincare metric on the unit disc and is nonincreasing under holomorphic mappings. Hence

$$
F_{C}^{\Omega}(P, \xi) \leq F_{S}^{\Omega}(P, \xi) \leq F_{K}^{\Omega}(P, \xi) .
$$


Finite type. We say the boundary of a domain in $\mathbb{C}^{n}$ is of finite type if the maximum tangency of the boundary with any one dimensional holomorphic variety is finite, that is,

$$
\sup \left\{\frac{v_{0}(\rho \circ \phi)}{v_{0}(\phi)}: \phi \in \mathbb{C}^{n}(\mathbb{D}), \phi(0)=P\right\}<\infty .
$$

For more details, see [Krantz 2001; D’Angelo 1993]. McNeal [1992] showed that the finite type condition of a boundary of a convex domain in $\mathbb{C}^{n}$ is same as the finite type condition with $\phi$ replaced with complex lines through $P$. So we introduced the notation $\Delta(\partial \Omega, P, \xi)$ in (1-2), which actually gives you the type in the direction $\xi$ if $\Omega$ is convex.

\section{Estimation on a convex domain}

Throughout this section we assume that $\Omega=\{\rho<0\} \Subset \mathbb{C}^{n}$ is a smoothly bounded convex domain, $P \in \partial \Omega, \xi \in T_{P}^{\mathbb{C}}(\partial \Omega)$ and the outward unit normal vector at $P$ is

$$
v=\frac{\nabla \rho(P)}{\|\nabla \rho(P)\|} .
$$

Let $P_{\delta}=P-\delta v$.

Lemma 1 [Bruna et al. 1988]. Let us define a set of functions on $\mathbb{R}$ as

$$
C(m, r) \equiv\left\{f(x)=a_{2} x^{2}+\cdots+a_{m} x^{m}: a_{i} \in \mathbb{R}, f^{\prime \prime}(x) \geq 0 \text { for all } x \in[0, r]\right\} .
$$

Then there exists a constant $C$ such that

$$
f(x) \geq C\left(\left|a_{2}\right| x^{2}+\cdots+\left|a_{m}\right| x^{m}\right),
$$

for all $f \in C(m, r)$ and all $x \in[0, r]$.

Proposition 2. Let $\Omega=\{\rho<0\} \Subset \mathbb{C}^{n}$ be a smoothly bounded convex domain, $P \in \partial \Omega$ and $v$ the unit outward real normal vector to $\partial \Omega$ at $P$ with $\|v\|=1$. Let $\xi \in T_{P}^{\mathbb{C}}(\partial \Omega),\|\xi\|=1$, and $\Delta(P, \partial \Omega, \xi)>2$. If we let

$$
R_{\xi}(\delta):=\sup \{|z|: P-\delta v+\xi z \in \Omega, z \in \mathbb{C}\},
$$

then

$$
R_{\xi}(\delta) \approx \delta^{1 / m}
$$

for $\delta>0$ sufficiently small.

Proof. We may assume

$$
P=0, \quad \nabla \rho(P)=(0, \ldots, 1) \quad \text { and } \quad \xi=(1,0, \ldots, 0) .
$$


Then near $P=0, \rho$ can be expressed as $\rho=\operatorname{Re} z_{n}+O\left(|z|^{2}\right)$ and, if we evaluate $\rho$ at $(0, \ldots, 0,-\delta)$ in the $z_{1}$-direction, we get

$$
\rho((\zeta, 0, \ldots, 0,-\delta))=-\delta+\sum_{\substack{p+q_{1}+q_{2}=2 \\ p \geq 1}}^{m-1} a_{p q_{1} q_{2}} \delta^{p} \zeta^{q_{1}} \bar{\zeta}^{q_{2}}+O\left(\left(\delta^{2}+|\zeta|^{2}\right)^{m / 2}\right) .
$$

Let $|\zeta|=c \delta^{1 / m}$. Then

$$
\begin{aligned}
\rho((\zeta, 0, \ldots, 0,-\delta)) & =-\delta+\sum_{\substack{p+q=2 \\
p \geq 1}}^{m-1} b_{p q} \delta^{p}\left(c \delta^{1 / m}\right)^{q}+O\left(\left(\delta^{2}+\left(c \delta^{1 / m}\right)^{2}\right)^{m / 2}\right) \\
& \leq-\delta+\sum_{\substack{p+q=2 \\
p \geq 1}}^{m-1} b_{p q} \delta^{p}\left(c \delta^{1 / m}\right)^{q}+C\left(\delta^{m}+c^{\prime} \delta\right)<0,
\end{aligned}
$$

for some constants $C$ and $c^{\prime}$. Hence, we get

$$
R_{\xi}(\delta) \gtrsim \delta^{1 / m}
$$

Next, we will show that, for a fixed $\epsilon \in\left(0, \frac{1}{m}\right)$, there does not exist a constant $c$ such that, for all sufficiently small $\delta$,

$$
\rho((\zeta, 0, \ldots, 0,-\delta))<0, \quad|\zeta|=c \delta^{(1 / m)-\epsilon} .
$$

Let $|\zeta|=c \delta^{(1 / m)-\epsilon}$ and look at the Taylor expansion:

$$
\begin{aligned}
& \rho((\zeta, 0, \ldots, 0,-\delta))= \\
& \quad-\delta+\sum_{\substack{p+q=2 \\
p \geq 1}}^{m} b_{p q} \delta^{p}\left(c \delta^{(1 / m)-\epsilon}\right)^{q}+b_{0 m}\left(c \delta^{(1 / m)-\epsilon}\right)^{m}+O\left(\left(\delta^{2}+\left(c \delta^{(1 / m)-\epsilon}\right)^{2}\right)^{(m+1) / 2}\right) .
\end{aligned}
$$

By Lemma 1, we get

$$
\begin{aligned}
\rho((\zeta, 0, \ldots, 0,-\delta)) & \geq-\delta+C\left(\sum_{\substack{p+q=2 \\
p \geq 1}}^{m}\left|b_{p q}\right| \delta^{p}\left(c \delta^{(1 / m)-\epsilon}\right)^{q}+\left|b_{0 m}\right|\left(c \delta^{(1 / m)-\epsilon}\right)^{m}\right) \\
& \geq-\delta+C\left|b_{0 m}\right|\left(c \delta^{(1 / m)-\epsilon}\right)^{m}-C^{\prime}\left(\delta^{m+1}+c^{\prime}\left(\delta^{(1 / m)-\epsilon}\right)^{m+1}\right) \\
& \left.=c^{\prime}\left(\delta^{(1 / m)-\epsilon}\right)^{m+1}\right) \\
& =C^{\prime \prime} \delta^{1-\epsilon m}-\delta-C^{\prime}\left(\delta^{m+1}+c^{\prime}\left(\delta^{(1 / m)-\epsilon}\right)^{m+1}\right)>0,
\end{aligned}
$$

for $\delta$ sufficiently small. Hence, we conclude that

$$
R_{\xi}(\delta) \approx \delta^{1 / m}
$$


To find the lower bound for the Sibony metric, we construct a plurisubharmonic function that satisfies the conditions of the definition and has a large Hessian in the $\xi$-direction. We could find such a plurisubharmonic function by modifying the construction of a plurisubharmonic function in [McNeal 1992].

Proposition 3. Suppose $\xi \in T_{P}^{\mathbb{C}}(\partial \Omega)$ and $\Delta(\partial \Omega, P, \xi)=m$. Then

$$
F\left(P_{\delta}, \xi\right) \gtrsim \frac{|\xi|}{\delta^{1 / m}}
$$

Proof. We may assume $\xi$ is the $z_{1}$-direction. We let the $\operatorname{Re} z_{1}$-direction be such that the distance from $P-\delta v$ to the boundary along $\operatorname{Re} z_{1}$ axis will be the greatest among all distances between $P-\delta v$ and the boundary along the $z_{1}$ axis, that is,

$$
\begin{aligned}
\sup \{r>0: \rho((r, 0, \ldots, 0,-\delta)) & \in \Omega\} \\
& =\sup \left\{r>0: \rho\left(\left(r e^{i \theta}, 0, \ldots, 0,-\delta\right)\right) \in \Omega, 0 \leq \theta<2 \pi\right\} .
\end{aligned}
$$

Let $R$ be such a distance

$$
R=\sup \{r>0: \rho((r, 0, \ldots, 0,-\delta)) \in \Omega\} .
$$

Then by Proposition 3, we know that

$$
R \approx \delta^{1 / m}
$$

Let $Q=(R, 0, \ldots, 0,-\delta)$. Now we will show that

$$
\frac{\partial \rho}{\partial z_{1}}(Q) \approx \delta^{1-(1 / m)} .
$$

using the technique in [McNeal 1992].

Consider the real tangent space to $\partial \Omega$ at $Q$ :

$\operatorname{Re}\left(\frac{\partial \rho}{\partial z_{1}}(Q)\left(z_{1}-R\right)+\frac{\partial \rho}{\partial z_{2}}(Q) z_{2}+\cdots+\frac{\partial \rho}{\partial z_{n-1}}(Q) z_{n-1}+\frac{\partial \rho}{\partial z_{n}}(Q)\left(z_{n}+\delta\right)\right)=0$.

Let $S$ be the intersection point between the above tangent space and the $\operatorname{Re} z_{n}$ axis, that is,

$$
\begin{aligned}
S=\{z: \operatorname{Re} & \left(\frac{\partial \rho}{\partial z_{1}}(Q)\left(z_{1}-R\right)+\frac{\partial \rho}{\partial z_{2}}(Q) z_{2}+\cdots\right. \\
& \left.\left.+\frac{\partial \rho}{\partial z_{n-1}}(Q) z_{n-1}+\frac{\partial \rho}{\partial z_{n}}(Q)\left(z_{n}+\delta\right)\right)=0\right\} \cap\{(0, \ldots, 0, x), x \in \mathbb{R}\} .
\end{aligned}
$$

If we let $S=(s, 0, \ldots, 0)$, then, by convexity, we have

$$
|S-P|=|(s, 0, \ldots, 0)-0|=s \geq 0 .
$$


Therefore, if we evaluate the tangent space at $S$, we get

$$
\operatorname{Re}\left(\frac{\partial \rho}{\partial z_{1}}(Q)(-R)+\frac{\partial \rho}{\partial z_{n}}(Q)(s+\delta)\right)=0 .
$$

Hence we have

$$
\left|\frac{\partial \rho}{\partial z_{1}}(Q)\right| R \geq\left|\operatorname{Re} \frac{\partial \rho}{\partial z_{1}}(Q) R\right|=\left|\frac{\partial \rho}{\partial z_{n}}(Q)\right|(s+\delta) \geq\left|\frac{\partial \rho}{\partial z_{n}}(Q)\right| \delta \approx \delta .
$$

Therefore,

$$
\left|\frac{\partial \rho}{\partial z_{1}}(Q)\right| \gtrsim \frac{\delta}{R} \approx \frac{\delta}{\delta^{1 / m}}=\delta^{1-(1 / m)} .
$$

To show the other direction, we look at the Taylor expansion of $\rho$ at 0 and evaluate it along the $z_{1}$-direction:

$$
\begin{aligned}
& \rho((z, 0, \ldots,-\delta))=-\delta+\sum_{\substack{p+q_{1}+q_{2}=2 \\
p \geq 1}}^{m} a_{p q_{1} q_{2}} \delta^{p} z^{q_{1}} \bar{z}^{q_{2}} \\
&+\sum_{r_{1}+r_{2}=m} a_{r_{1} r_{2}} z^{r_{1}} \bar{z}^{r_{2}}+O\left(\left(\delta^{2}+|z|^{2}\right)^{(m+1) / 2}\right) .
\end{aligned}
$$

Differentiating along the $z_{1}$-direction, we get

$$
\frac{\partial \rho}{\partial z_{1}}(Q)=\sum_{\substack{p+q=2 \\ p \geq 1}}^{m} b_{p q} \delta^{p} R^{q-1}+b_{m} R^{m-1}+O\left(\left(\delta^{2}+|z|^{2}\right)^{m / 2}\right) .
$$

Since $R \leq C \delta^{1 / m}$, we get

$$
\begin{aligned}
\left|\frac{\partial \rho}{\partial z_{1}}(Q)\right| \lesssim \sum_{\substack{p+q=2 \\
p \geq 1}}^{m}\left|b_{p q}\right| C^{q-1} \delta^{p+(q-1) / m} \\
\quad+\left|b_{m}\right| C^{m-1} \delta^{1-(1 / m)}+O\left(\delta^{m}+C^{m} \delta\right) \lesssim \delta^{1-(1 / m)} .
\end{aligned}
$$

By (3-1) and (3-2), we get

$$
\left|\frac{\partial \rho}{\partial z_{1}}(Q)\right| \approx \delta^{1-(1 / m)} .
$$

Now we construct a candidate plurisubharmonic function for the Sibony metric $F_{S}(P-\delta v, \xi)$. Let

$$
f=\frac{1}{\delta}\left(\frac{\partial \rho}{\partial z_{1}}(Q) z_{1}+\cdots+\frac{\partial \rho}{\partial z_{n-1}}(Q) z_{n-1}+\frac{\partial \rho}{\partial z_{n}}(Q)\left(z_{n}+\delta\right)\right)
$$

and define

$$
F_{N}=f+\frac{1}{2 !} f^{2}+\cdots+\frac{1}{N !} f^{N}
$$


where the number $N$ will be chosen later. If we let

$$
G_{N}=\left|F_{N}\right|^{2}
$$

then $G_{N}$ satisfies the following properties: $G_{N}(P-\delta v)=0, \log G_{N}$ is plurisubharmonic on $\Omega$, and

$$
\frac{\partial^{2} G_{N}}{\partial z_{1} \partial \bar{z}_{1}}(P-\delta v)=\frac{1}{\delta^{2}}\left|\frac{\partial \rho}{\partial z_{1}}(Q)\right|^{2} \approx \frac{\delta^{2-(2 / m)}}{\delta^{2}}=\left(\frac{1}{\delta^{1 / m}}\right)^{2} .
$$

Hence, if we can show that $G_{N}$ is bounded on $\Omega$, then we can conclude that

$$
F_{S}^{\Omega}\left(P_{\delta}, \xi\right) \gtrsim \frac{|\xi|}{\delta^{1 / m}}
$$

Now we prove that $G_{N}$ is bounded and has an upper bound independent of $\delta$. Since

$$
1+f+\frac{1}{2 !} f^{2}+\cdots+\frac{1}{k !} f^{k}+\cdots=\exp f
$$

we may find $N$ such that

$$
\left|1+F_{N}(z)-\exp f(z)\right|<1, \quad \text { for all } z \in \Omega .
$$

Therefore,

$$
\left|F_{N}\right|<1+|\exp f-1| \leq 2+e^{\operatorname{Re} f} .
$$

Since $\operatorname{Re} f=0$ defines a hyperplane and $\operatorname{Re} f$ changes sign at $\operatorname{Re} f=0$, we may assume that $\operatorname{Re} f>0$ near the boundary and negative elsewhere. Then

$$
e^{\operatorname{Re} f} \leq 1
$$

outside a small neighborhood of $P$. Therefore we have

$$
\operatorname{Re} f \leq \operatorname{Re} f(Q)=\frac{1}{\delta} \operatorname{Re}\left(\frac{\partial \rho}{\partial z_{1}}(Q) R\right) \lesssim \frac{1}{\delta} \delta^{1-(1 / m)} \delta^{1 / m}=1 .
$$

Hence $G_{N}$ is uniformly bounded for all $\delta$.

\section{Proposition 4.}

$$
F\left(P_{\delta}, v\right) \geq \frac{1}{6 \delta}
$$

Proof. Let

$$
\rho(z)=2 \operatorname{Re} z_{n}+O\left(|z|^{2}\right) .
$$

Since $\Omega$ is convex, we see that $\Omega \subset\left\{\operatorname{Re} z_{n}<0\right\}$. Let us look at the function

$$
u(z)=\frac{1}{9}\left|\frac{z_{n}+\delta}{z_{n}-\delta}\right|^{2} .
$$


Since $\operatorname{Re} z_{n}<0$ for all $z \in \Omega$, the function inside the absolute value sign is holomorphic on $\Omega$. Hence $\log u$ is plurisubharmonic on $\Omega$. And $u(P-\delta v)=0$. We also have

$$
\left|\frac{z_{n}+\delta}{z_{n}-\delta}\right| \leq 1+\left|\frac{2 \delta}{z_{n}-\delta}\right| \leq 1+\frac{2 \delta}{\delta}=3, \quad z \in \Omega,
$$

since

$$
\left|z_{n}-\delta\right| \geq\left|\operatorname{Re} z_{n}-\delta\right|=\left|\operatorname{Re} z_{n}\right|+\delta \geq \delta, \quad \text { for all } z \in \Omega \text {. }
$$

Hence $0 \leq u(z) \leq 1$ on $\Omega$. Finally,

$$
\left(\frac{\partial^{2} u\left(P_{\delta}\right)}{\partial z_{n} \partial \bar{z}_{n}}\right)^{1 / 2}=\left(\frac{1}{9} \frac{1}{4 \delta^{2}}\right)^{1 / 2}=\frac{1}{6 \delta} .
$$

Hence Theorem 1 is proved by Proposition 3 and Proposition 4.

Proof of Theorem 2. We use the same function $u(z)$

$$
u(z)=\frac{1}{9}\left|\frac{z_{n}+\delta}{z_{n}-\delta}\right|^{2}
$$

as in (3-3). Then we have that

$$
F_{S}\left(P_{\delta}, X\right) \geq(\partial \bar{\partial} u(X, \bar{X}))^{1 / 2}=\frac{|a|}{6 \delta} .
$$

\section{A modification of the Sibony metric}

In this section, we discuss a possible modification of the Sibony metric.

Definition 2 (Plurisubharmonic metric). Let $\Omega \subset \mathbb{C}^{n}$ be a domain and $P \in \Omega$, $\xi \in \mathbb{C}^{n}$. We define a set of functions $B_{\Omega}(P, \xi)$ by the condition that $u \in B_{\Omega}(P, \xi)$ if and only if

(1) $u$ is $C^{2}$ near $P$;

(2) $u(P)=0$;

(3) there exists a holomorphic disc $f: \mathbb{D} \rightarrow \bar{\Omega}$ such that

$$
f(0)=P, \quad f^{\prime}(0)=\frac{\xi}{F_{K}^{\Omega}(P, \xi)},
$$

and $u$ satisfies

(a) $0 \leq u \circ f(z) \leq 1$, for all $z \in \mathbb{D}$ and (b) $\frac{u \circ f(z)}{|z|^{2}}$ is subharmonic on $\mathbb{D}$.

We define the plurisubharmonic metric $F_{\Omega}^{P}$ at $P \in \Omega$ in the direction $\xi \in \mathbb{C}^{n}$ as

$$
F_{P}^{\Omega}(P, \xi) \equiv \sup _{u \in B_{\Omega}(P, \xi)}\left(\sum_{i, j=1}^{n} \frac{\partial^{2} u}{\partial z_{i} \partial \bar{z}_{j}}(P) \xi_{i} \bar{\xi}_{j}\right)^{1 / 2} .
$$


Proposition 5. If $\Omega \Subset \mathbb{C}^{n}$ is a pseudoconvex domain and $P \in \Omega$ and $\xi \in \mathbb{C}^{n}$, then

$$
F_{S}^{\Omega}(P, \xi) \leq F_{P}^{\Omega}(P, \xi) .
$$

Proof. It is enough to show that the collection of candidate functions for the Sibony metric is a subset of collection of candidate functions for the plurisubharmonic metric, that is, $A_{\Omega}(P) \subset B_{\Omega}(P, \xi)$.

If $u \in A_{\Omega}(P)$, then, as stated in Definition $1, u$ is $C^{2}$ near $P, u(P)=0,0 \leq$ $u(z) \leq 1$ for all $z \in \Omega$ and $\log u$ is plurisubharmonic on $\Omega$. We need to show that $u$ satisfies the conditions of Definition 2 .

Since $\Omega$ is pseudoconvex, we can find an extremal disc $f \in \Omega(\mathbb{D})$ such that

$$
f(0)=P \quad \text { and } \quad f^{\prime}(0)=\frac{\xi}{F_{K}^{\Omega}(P, \xi)} .
$$

Hence, since $0 \leq u \leq 1$ on $\Omega$, we get $0 \leq u \circ f(z) \leq 1$ for all $z \in \mathbb{D}$. We know that $\log u$ is plurisubharmonic on $\Omega$. Therefore, $\log u \circ f$ is subharmonic on $\mathbb{D}$. Since $\log |z|$ is harmonic, $\log u \circ f-\log |z|^{2}$ is subharmonic on $\mathbb{D}$. Taking the exponential, we see that

$$
\frac{u \circ f(z)}{|z|^{2}}
$$

is subharmonic on $\mathbb{D}$.

Next we will show that the metric $F_{P}^{\Omega}$ is invariant under biholomorphic mappings. This is connected to the fact that the Kobayashi metric is invariant under biholomorphic mappings.

Proposition 6. The plurisubharmonic metric is invariant under biholomorphic mappings.

Proof. Let $\Omega_{1}, \Omega_{2} \subset \mathbb{C}^{n}, P \in \Omega_{1}$ and $\xi \in \mathbb{C}^{n}$. Suppose that $\Phi: \Omega_{1} \rightarrow \Omega_{2}$ is a biholomorphic mapping. We will show that

$$
u \circ \Phi^{-1} \in B_{\Omega_{2}}\left(\Phi(P), \Phi_{*}(P) \xi\right), \quad \text { for all } u \in B_{\Omega_{1}}(P, \xi),
$$

where $B_{\Omega}(P, \xi)$ is the set of functions that satisfy the conditions of Definition 2. Since $\Phi$ is a biholomorphic mapping, $u \circ \Phi^{-1}$ is $C^{2}$ near $\Phi(P)$ and

$$
u \circ \Phi^{-1}(\Phi(P))=u(P)=0 .
$$

Thus the first two conditions are satisfied. Now suppose that $f: \mathbb{D} \rightarrow \bar{\Omega}_{1}$ is a holomorphic curve that satisfies the conditions of Definition 2 for $F_{P}^{\Omega_{1}}(P, \xi)$. If 
we let $g(z)=\Phi \circ f(z)$, then $g(0)=\Phi(f(0))=\Phi(P)$ and

$$
\begin{aligned}
g^{\prime}(0) & =\operatorname{Jac} \Phi(P) f^{\prime}(0)=\operatorname{Jac} \Phi(P) \frac{\xi}{F_{\Omega_{1}}^{K}(P, \xi)}=\frac{\Phi_{*}(P) \xi}{F_{\Omega_{1}}^{K}(P, \xi)} \\
& =\frac{\Phi_{*}(P) \xi}{F_{\Omega_{2}}^{K}\left(\Phi(P), \Phi_{*}(P) \xi\right)} .
\end{aligned}
$$

The last equality holds since the Kobayashi metric is invariant under $\Phi$.

Now we want to show that $u \circ \Phi^{-1}$ satisfies the conditions on the holomorphic curve $\Phi \circ f$. But this is rather straightforward since $f$ was chosen to be the holomorphic curve on which the function $u$ satisfies the conditions of Definition 2 and

$$
u \circ \Phi^{-1} \circ \Phi \circ f(z)=u \circ f(z) .
$$

Now we want to prove that the plurisubharmonic metric coincides with the Poincaré metric on the unit disc in $\mathbb{C}$.

Proposition 7. The plurisubharmonic metric coincides with the Poincaré metric on the unit disc in $\mathbb{C}$, that is,

$$
F_{P}(0, \xi)=P_{\mathbb{D}}(0, \xi)=|\xi| .
$$

Proof. By the following lemma, we know that $F_{P}(0, \xi) \leq|\xi|$. Also, since $u(z)=$ $|z|^{2}$ satisfies the conditions of being a candidate function, we get

$$
F_{P}(0, \xi)=|\xi| \text {. }
$$

Lemma 2. If $u: \mathbb{D} \rightarrow \mathbb{R}$ satisfies

(1) $u(0)=0$, $u$ is $C^{2}$ near 0 ;

(2) $0 \leq u \leq 1$ on $\mathbb{D}$;

(3) $u(z) /|z|^{2}$ is subharmonic on $\mathbb{D}$,

then

$$
\frac{\partial^{2} u(0)}{\partial z \partial \bar{z}} \leq 1
$$

Proof. Since $u$ is $C^{2}$ near 0 and has a minimum at 0 , the first order derivatives of $u$ at 0 is 0 . Hence the Taylor expansion near 0 becomes

$$
u(z)=a|z|^{2}+\operatorname{Re} b z^{2}+O\left(|z|^{3}\right), \quad a \in \mathbb{R}, b \in \mathbb{C} .
$$

Let $z=|z| e^{i \theta}$. Then

$$
\frac{u(z)}{|z|^{2}}=a+\operatorname{Re}\left(b e^{i \theta}\right)+O(|z|)
$$


Since $\frac{u(z)}{|z|^{2}}$ is subharmonic on $\mathbb{D}$, by the maximum principle we get

$$
\left.\frac{u(z)}{|z|^{2}}\right|_{z=0} \leq\left.\frac{u(z)}{|z|^{2}}\right|_{|z|=1}=\left.u(z)\right|_{|z|=1} \leq 1 .
$$

Therefore,

$$
\left.\frac{u(z)}{|z|^{2}}\right|_{z=0}=a+\operatorname{Re}\left(b e^{2 i \theta}\right) \leq 1
$$

Choose $\theta_{0}$ such that $\operatorname{Re}\left(b e^{2 i \theta_{0}}\right) \geq 0$. We get

$$
a \leq 1-\operatorname{Re}\left(b e^{2 i \theta_{0}}\right) \leq 1 .
$$

Proposition 8. The plurisubharmonic metric is less than or equal to the Kobayashi metric, that is,

$$
F_{P}^{\Omega}(P, \xi) \leq F_{K}^{\Omega}(P, \xi) .
$$

Proof. Let $u$ be the candidate function and $f$ be the curve that has the derivative at the base point of the same size as the inverse of the Kobayashi metric in the $\xi$ direction such that $0 \leq u \circ f \leq 1$ on $\mathbb{D}$ and $\frac{u \circ f(z)}{|z|^{2}}$ is subharmonic on $\mathbb{D}$. Then by Lemma 2, we get

$$
\frac{\partial^{2} u \circ f(0)}{\partial z \partial \bar{z}} \leq 1
$$

Rewriting the left hand side of the above inequality, we get

$$
\frac{\partial^{2} u \circ f(0)}{\partial z \partial \bar{z}}=\sum_{j, k} \frac{\partial^{2} u(P)}{\partial \xi_{j} \partial \bar{\xi}_{k}}\left(f^{\prime}(0)\right)_{j}\left(\bar{f}^{\prime}(0)\right)_{k}=\frac{1}{\left(F_{K}(P, \xi)\right)^{2}} \sum_{j, k} \frac{\partial^{2} u(P)}{\partial \xi_{j} \partial \bar{\xi}_{k}} \xi_{j} \bar{\xi}_{k} .
$$

Hence

$$
\left(\sum_{j, k} \frac{\partial^{2} u(P)}{\partial \xi_{j} \partial \bar{\xi}_{k}} \xi_{j} \bar{\xi}_{k}\right)^{1 / 2} \leq F_{K}(P, \xi)
$$

Therefore,

$$
F_{P}(P, \xi) \leq F_{K}(P, \xi)
$$

\section{References}

[Bruna et al. 1988] J. Bruna, A. Nagel, and S. Wainger, "Convex hypersurfaces and Fourier transforms", Ann. of Math. (2) 127:2 (1988), 333-365. MR 89d:42023 Zbl 0666.42010

[Catlin 1989] D. W. Catlin, "Estimates of invariant metrics on pseudoconvex domains of dimension two", Math. Z. 200:3 (1989), 429-466. MR 90e:32029 Zbl 0661.32030

[D'Angelo 1993] J. P. D'Angelo, Several complex variables and the geometry of real hypersurfaces, Studies in Advanced Mathematics, CRC Press, Boca Raton, FL, 1993. MR 94i:32022 Zbl 0854.32001

[Graham 1975] I. Graham, "Boundary behavior of the Carathéodory and Kobayashi metrics on strongly pseudoconvex domains in $C^{n}$ with smooth boundary", Trans. Amer. Math. Soc. 207 (1975), 219-240. MR 51 \#8468 Zbl 0305.32011 
[Krantz 2001] S. G. Krantz, Function theory of several complex variables, AMS Chelsea Publishing, Providence, RI, 2001. Reprint of the 1992 edition. MR 2002e:32001 Zbl 1087.32001

[Lee 2007] L. Lee, Asymptotic behavior of invariant metrics, thesis, Washington Univ. in St. Louis, 2007.

[Lempert 1981] L. Lempert, "La métrique de Kobayashi et la représentation des domaines sur la boule”, Bull. Soc. Math. France 109:4 (1981), 427-474. MR 84d:32036 Zbl 0492.32025

[McNeal 1992] J. D. McNeal, “Convex domains of finite type”, J. Funct. Anal. 108:2 (1992), 361373. MR 93h:32020 Zbl 0777.31007

Received December 21, 2007. Revised April 28, 2008.

LINA LEE

DEPARTMENT OF MATHEMATiCS

THE UNIVERSITY OF MICHIGAN

EAST Hall, 530 ChURCH St.

ANN ARBOR, MI 48109

UNITED STATES

linalee@umich.edu 